\title{
Pneumatic supply system parameter optimization for soft actuators
}

\author{
Sagar Joshi and Jamie Paik*
}

\begin{abstract}
Soft actuators using pressurized air are being widely used due to their inherent compliance, conformability and customizability. These actuators are powered and controlled by pneumatic supply systems (PSSs) consisting of components such as compressors, valves, tubing and reservoirs. Regardless of the choice of actuator, the PSS critically affects overall performance of soft robots because it governs the soft actuator pressure dynamics and thereby, the general dynamic behaviour. While selecting and controlling PSS components for meeting desired soft actuator performance, specifications such as PSS mass, volume and duration of operation must also be considered. Currently, there is no comprehensive study on PSS optimization for meeting dynamic performance and PSS specifications, due to limited understanding of soft actuator pressure dynamics, large solution space for PSSs, and variability in soft actuators. By considering critical parameters of PSS and soft actuators, we introduce and demonstrate PSS parameter optimization. We propose a normalized model for soft actuator pressure dynamics and quantify the relationship between PSS parameters, soft actuator design parameters and dynamic performance metrics of rise time, fall time and actuation frequency. Using these results, we optimally select and control PSS components to meet desired soft actuator performance for a soft exosuit, while minimizing mass of selected components. The measured pressure response with this prototype agrees well with simulations, with root mean square errors under $5.2 \%$. This work is a step towards furthering the scope of soft robotics, as it enables PSS optimization, for meeting the desired soft actuator performance while also addressing PSS specifications.
\end{abstract}

\section{INTRODUCTION}

Soft robotics [1], [2] provides customizable solutions to a range of applications such as wearable devices [3]-[9], mobile robots [10]-[14] and grippers [2], [15], [16]; due to unique characteristics such as compliance, conformability, and versatility. A significant number of soft robots generate motion via soft pneumatic actuators or SPAs (Fig. 1B) powered by pneumatic supply systems (PSSs) [4]-[13], [17][21]. PSSs (Fig. 11A) typically consist of three main components: source for generating pressurized air, pneumatic line for connection, and valves for controlling flow direction [22]. By virtue of the pneumatic flow, PSSs govern the soft actuator pressure dynamics, and thus the overall soft robot dynamic behaviour [1], [2], [22]. In order to meet dynamic performance specifications such as rise time, fall time or actuation

S. Joshi and J. Paik are with the Reconfigurable Robotics Lab (RRL), Swiss Federal Institute of Technology Lausanne, Lausanne 1015, Switzerland (email: sagar.joshi@epfl.ch; jamie.paik@epfl.ch) 
frequency, PSSs have to be tailored, i.e. the PSS components and their control parameters such as source pressure, valve duty cycle and compressor duty cycle must be selected carefully. Regardless of the soft actuator used, improper selection or control of PSS components can lead to either too slow or too fast dynamic response, or can even damage the soft actuator.

Accordingly, researchers have studied some aspects of PSSs for soft robotic applications. Wehner et al. [22] compared portability and relative merits and demerits of different choices for the pneumatic source such as battery powered compressors, liquid $\mathrm{CO}_{2}$, and peroxide decomposition. However, this study does not include the role of valve, pneumatic lines and soft actuator design parameters such as internal volume, operating pressure and maximum expansion. Additionally, it does not investigate soft actuator dynamic behaviour. In another study [23], researchers heuristically tuned source pressure and valve duty cycle to increase maximum actuation frequency from $2 \mathrm{~Hz}$ to $4 \mathrm{~Hz}$. While effective for tuning PSS control parameters, such a heuristic approach is insufficient for PSS component selection. Furthermore, it cannot predict how dynamic behaviour will be affected if the soft actuator or a PSS component is changed. By employing finite elemental analysis (FEA), Marchese et al. [13] calculated the source pressure required to achieve high speed inflation response in a soft robotic fish. However, their results were limited to the specific application and do not lead to a general understanding of PSSs' influence on the soft actuator dynamic behaviour. As of yet, the relationship between PSS components, soft actuator design parameters and dynamic performance is not well-quantified.

While there are numerous studies on soft actuator kinematics, deformation and force interactions using analytical modelling [14], [24]-[26], rigid-kinematic approximations [27]-[29], or FEA [30], [31], soft actuator pressure dynamics are relatively less explored. The major reasons limiting study of soft actuator pressure dynamics are: (i) non-linear nature of pneumatic flow, (ii) non-linear nature of soft actuator behaviour due to material properties, design geometry and large deformations, (iii) large variability in soft actuator design with respect to design, materials, and operating pressures [1], [32], and (iv) large variability in PSSs with respect to components and their control parameters. As a result, soft actuator dynamic response is most commonly modelled by fitting the experimentally measured response to first or second order linear systems [6], [12]-[14], [29], [33], [34]. Such models do not relay information about the underlying flow mechanics, and are insufficient for customizing PSSs to meet performance requirements. In addition to performance, applications often dictate specifications on the PSS in terms of its mass, volume, tubing length and duration of operation; and a trade-off has to be made in PSS design, as improved dynamic performance necessitates a bulkier PSS as seen in Fig. 1C [22]. Currently there is no systematic method for PSS component selection and control to simultaneously meet specifications of dynamic performance and the PSS. 
By studying the effect of critical parameters of PSSs and soft actuators on dynamic performance, here, we introduce parameter optimization. We first propose a comprehensive normalized model for soft actuator pressure dynamics, considering the effect of source, valve and pneumatic line. Using this model, we create a direct relationship between PSS parameters, soft actuator design parameters and dynamic performance metrics of rise time, fall time and maximum actuation frequency. We apply results from this study and design and test a portable PSS for a wearable robot, that meets desired dynamic performance while minimizing mass of selected components. In addition to improving the understanding soft actuator pressure dynamics, this work enables optimal design through selection and control of PSS components to simultaneously address specifications of PSS and soft actuator performance. The main contributions of this work are:

- A comprehensive model for soft actuator pressure dynamics.

- Investigation of the impact of PSS parameters and soft actuator design parameters on actuators' dynamic performance.

- Validation of PSS parameter optimization via novel soft exosuit prototype design and testing.

\section{PROPOSED APPROACH FOR PSS PARAMETER OPTIMIZATION}

Existing PSSs in industry and academia are found prominently in two configurations depending on the source used, as shown in Fig. 2 .

- Configuration A: This includes PSSs with a pressure regulator at the outlet of either large off-board compressors, high pressure gas cylinders $(20$ or $30 \mathrm{MPa} G)$ or liquid $C O_{2}$ cylinders $(5.5 \mathrm{MPa} G)$ as the source (Fig. 2]A) [2], [13], [23]. Assuming perfect regulation, we model these as constant pressure PSSs.

- Configuration B: This includes PSSs consisting of an on-board compressor and a reservoir as the source (Fig. 2B) [10], [15], [35]. We model these as constant flow PSSs.

In order to optimally select and control PSS components, we first define a component library by noting the set of available options for the source, valve and pneumatic line. For the source, we consider the two configurations described above. For the valve, we consider solenoid valves and for pneumatic line we consider tubing and fittings; because these are used most prominently. We then identify critical parameters of these PSS components affecting pneumatic flow, and classify them into two types as follows:

- flow parameters, $f$ : These include non-varying pneumatic parameters such as internal volumes of reservoir, tubing and fittings; and flow capacities of regulators, valves, compressors, tubing and fittings. These parameters form a discrete set of values. 
- control parameters, $c$ : These include actively controlled parameters such as source pressure, and duty cycles of compressor and valves; which can take continuously varying values.

Table 1 lists the flow and control parameters considered for this study. The PSS component library thus contains the list of all available components along with their corresponding parameters: $f$ and $c$. Next, we identify the soft actuator design parameters affecting its pressure dynamics, and denote them as $\alpha$ : operating pressure, volume and extent of deformation; as shown in Table I.

Lastly, we categorize design requirements as:

- Dynamic performance specifications: These include the soft actuator fall time, rise time and maximum actuation frequency.

- PSS specifications: These include the PSS volume, mass, tubing length, number of outputs and total air delivery.

For optimizing PSSs, the relationship between PSS parameters, soft actuator design parameters and the above specifications must be quantified. In the subsequent sections, we develop a model for soft actuator pressure dynamics, and simulate its pressure response while varying $f, c$ and $\alpha$, to create a direct relationship between these parameters and dynamic performance metrics. Using the mapping results, we can optimally select and control the PSS components from the component library for simultaneously meeting specifications of dynamic performance and PSS.

\section{MODELLING PRESSURE AND FLOW DYNAMICS}

In order to study pressure dynamics of soft actuators systematically, we use existing established models for air flow through PSS [36]-[41]. In addition, we propose a new quasi-static pressure-volume relationship for modelling soft actuator expansion. The commonly used assumptions in pneumatic studies [36]-[41] are as follows: (i) there is no air leakage; (ii) air follows Ideal gas equations; (iii) the ambient is at standard pressure and temperature, $P_{0}=0.1 \mathrm{MPa} A, T_{0}=293.15 \mathrm{~K}$; (iv) air compression and expansion occurs isothermally at $T_{0}$; (v) internal pressure for pressurized volumes at any instant is uniform, i.e. a lumped model. We apply the above assumptions in our study.

\section{A. Modelling flow through PSS}

As described in Fig. 2, we classify PSSs into two types based on the source used. For constant pressure PSSs (Fig. 2A), we have

$$
\dot{P}_{s r c}=0
$$


For constant flow PSSs (Fig. 2B), $P_{\text {src }}$ is affected by inflow from the compressor and outflow to the soft actuator, $Q_{s p a}$, where spa stands for soft pneumatic actuator. Applying Ideal gas equations, the pressure dynamics of the reservoir are given by (Eq. 2):

$$
\dot{P}_{\text {src }}=\frac{P_{0}}{V_{\text {res }}}\left(Q_{\text {com }}-\operatorname{pos}\left(Q_{\text {spa }}\right)\right)
$$

where $P_{s r c}$ is the instantaneous reservoir pressure; $V_{\text {res }}$ is the reservoir volume including dead volume of tubing and fittings between compressor and valve; $Q_{c o m}$ is the instantaneous mass flow output of the compressor expressed in standard L/s. pos $\left(Q_{s p a}\right)$ is the positive part of mass flow to soft actuator, i.e. from reservoir to soft actuator.

During soft actuator inflation and deflation, air flows through the valve, tubing and fittings. We model this flow using ISO 6358 [40], [42], which describes flow through a component using two parameters, sonic conductance $C$ and critical ratio $b$ (Eq. S3, S4] [42]. We find the combined effect of tubing, fitting and valves by calculating the equivalent $C_{e q}$ and $b_{e q}$, using $C$ and $b$ of each and applying appropriate relations for series and parallel combinations (Eq. S5 - S10).

\section{B. Modelling soft actuator pressure dynamics}

We model the soft actuator as a chamber with variable internal volume, $V_{s p a}$. Similar to Eq. 2, we can express its pressure dynamics as:

$$
\dot{P}_{s p a}=\frac{P_{0}}{V_{s p a}}\left(Q_{s p a}\right)-\frac{P_{s p a} \dot{V}_{s p a}}{V_{s p a}}
$$

where $P_{s p a}$ is the instantaneous soft actuator pressure; $V_{s p a}$ is the soft actuator internal volume including dead volume of tubing and fittings between valve and soft actuator; and $Q_{\text {spa }}$ is the mass flow to the soft actuator expressed in standard L/s.

$V_{\text {spa }}$ is governed by $P_{s p a}$, soft actuator design, external forces and inertial forces. Neglecting effect of the external and inertial forces, we express the freely expanding/contracting soft actuator volume quasistatically as a function of its pressure. While a general polynomial relation: $V_{s p a}=v\left(P_{s p a}\right)$ can be estimated from experimental characterization, we model a linear relationship based on [13], [23], [27], [32], [43]:

$$
V_{s p a}=V_{s p a}^{0}\left(1+K_{e x p}\left(P_{s p a}-P_{0}\right)\right)
$$

where we define $K_{e x p}$ as a constant relating $P_{s p a}$ to $V_{s p a}$; and $V_{s p a}^{0}$ is the volume of actuator at $P_{s p a}=P_{0}$.

The soft actuator pressure dynamics, while implicitly considering internal volume dynamics, are then described by:

$$
\dot{P}_{s p a}=\frac{P_{0} Q_{s p a}}{V_{s p a}^{0}\left(1+K_{\text {exp }}\left(2 P_{s p a}-P_{0}\right)\right)}
$$




\section{Normalization}

We normalize soft actuator and PSS pressure dynamics with soft actuator internal volume and the equivalent sonic conductance. We first define normalized sonic conductance as:

$$
\bar{C}=\frac{C_{e q}}{V_{s p a}^{0}}
$$

For constant flow PSSs, we additionally define normalized reservoir volume, $\bar{V}$, and compressor output flow, $\bar{Q}$ :

$$
\bar{V}=\frac{V_{r e s}}{V_{s p a}^{0}}, \quad \bar{Q}=\frac{Q_{c o m}}{V_{s p a}^{0}}
$$

Using the above, the normalized soft actuator pressure dynamics are given by Eq. S3, S4, S13, S14, S15, and S16. For constant pressure PSSs, the normalized dynamics depend on $P_{s r c}, P_{s p a}, K_{\text {exp }}$ and $b_{e q}$; whereas for constant flow PSSs, they additionally depend on $\bar{Q}$ and $\bar{V}$. The effect of $V_{s p a}^{0}$ and $\bar{C}$ is seen as scaling of the dynamics in magnitude and time respectively.

\section{GENERATING MAPPING FROM PSS PARAMETERS AND SOFT ACTUATOR PROPERTIES TO DYNAMIC PERFORMANCE}

Here, we simulate the normalized soft actuator pressure dynamics and use the predicted pressure response to quantify the relationship between dynamic performance and the parameters $f, c$ and $\alpha$, listed in Table I. We quantify soft actuator dynamic performance using the following metrics:

- $\boldsymbol{T}_{\text {fall }}$ : Time required for $P_{s p a}$ to drop from $98-2 \%$ of $P_{o p}^{g}$

- $\boldsymbol{T}_{\text {rise }}$ : Time required for $P_{s p a}$ to rise from $2-98 \%$ of $P_{o p}^{g}$

- $f_{m a x}$ : Maximum frequency at which soft actuator can be cyclically inflated at deflated between $2 \%$ and $98 \%$ of $P_{o p}^{g}$

where $P_{o p}^{g}$ is the soft actuator operating pressure, $P_{o p}$, expressed in gauge $\left(P_{o p}^{g}=P_{o p}-P_{0}\right)$; and $P_{0} \leq$ $P_{s p a} \leq P_{o p}$

\section{A. Methods}

1) Fall time and rise time: We simulated soft actuator pressure response of inflation and deflation between $0-0.2 \mathrm{MPa} G$, through a flow path of $\bar{C}=10 V_{\text {spa }}^{0} / \mathrm{s} / \mathrm{MPa}$. We carried out the simulations at a sampling rate of $10 \mathrm{kHz}$, for the following set of parameters:

a) $P_{s r c}: 0.2-0.6 \mathrm{MPa} G$, in steps of $0.025 \mathrm{MPa}$

b) $K_{\text {exp }}: 0-5 V_{s p a}^{0} / \mathrm{MPa}$, in steps of $0.5 V_{s p a}^{0} / \mathrm{MPa}$

c) $b_{e q}: 0.1-1$, in steps of 0.1

For constant flow PSSs, we further carried out simulations for: 
(d) $\bar{Q} / \bar{C}: 0.05-0.5 \mathrm{MPa}$ in steps of $0.05 \mathrm{MPa}$

(e) $\bar{V}$ : In six values ranging from 0.1-24 $V_{\text {spa }}^{0}$, as shown in Fig. 5 . $\bar{V}=0.1$ represents constant flow PSSs without a reservoir. We chose this small but non-zero value to account for internal dead volume of tubing and fittings.

By linear interpolation of the pressure response data, we calculated $T_{\text {fall }}$ and $T_{\text {rise }}$ for different operating pressures $P_{o p}: 0.005-0.2 \mathrm{MPa} G$, in steps of $0.005 \mathrm{MPa}$.

2) Maximum actuation frequency: For constant pressure PSSs, we calculated $f_{\max }$ by using results from $T_{\text {fall }}$ and $T_{\text {rise }}$. For constant flow PSSs, we used principle of conservation of mass to calculate $f_{\text {max }}$.

\section{B. Results}

1) Fall time, $T_{\text {fall }}:$ As air flows from the soft actuator to the ambient, soft actuator pressure dynamics do not depend on type of source, i.e. they are unaffected by $P_{s r c}, \bar{Q}, \bar{V}$ and $D_{C} . T_{\text {fall }}$ for different $P_{o p}$, $K_{e x p}$ and $b_{e q}$ are shown in Fig. 3. The labels depict the fall time scaled by $\bar{C}$. As seen from the figure, fall time increases with increasing $P_{o p}, K_{e x p}$ and decreases with $b_{e q}$ and $\bar{C}$.

2) Rise time, $T_{\text {rise }}$ : As air flows from the source to the soft actuator, constant pressure PSSs and constant flow PSSs exhibit different behaviour.

a) Constant pressure PSSs: Fig. 4 shows rise time for different $P_{s r c}, K_{e x p}, b_{e q}$ and $P_{o p}$. The labels on each curve depict the rise time scaled by $\bar{C}$. As seen from the figures, $T_{\text {rise }}$ increases with increasing $P_{o p}, K_{e x p}$ and decreases with $P_{s r c}, \bar{C}$ and $b_{e q}$.

b) Constant flow PSSs: Fig. 5 shows $T_{\text {rise }}$ of constant flow PSSs for varying $P_{s r c}, \bar{Q}$ and $\bar{V}$, for $P_{o p}=$ $0.2 \mathrm{MPa} G, K_{\text {exp }}=1.51 V_{\text {spa }}^{0} / M P a$ and $b_{e q}=0.49$. As seen from the figure, for certain pressures and reservoir volumes, $T_{\text {rise }}$ for constant flow PSSs is similar to that of constant pressure PSSs (dotted curve). This occurs because $P_{s r c}$ does not vary much during inflation, leading to a quasi-constant source pressure. However, at other values of $P_{s r c}$ and $\bar{V}, T_{\text {rise }}$ increases significantly. This occurs when $P_{s r c}$ drops below $P_{o p}$ during inflation, and so the compressor has to pressurize both reservoir and actuator. The point at which this occurs can be found using Fig. 5, or alternatively, by using conservation of mass, as described below.

Soft actuator pressure and normalized volume before inflation is $P_{0}$ and 1 respectively. After inflation, they become $P_{o p}$ and $1+K_{e x p}\left(P_{o p}-P_{0}\right)$. For the reservoir with volume $\bar{V}$, let pressure before and after inflation be $P_{s r c}$ and $P_{s r c}^{\prime}$ respectively. The air entering through the compressor is $P_{0} \bar{Q} T_{\text {rise }}$. In the limiting condition, $P_{s r c}$ drops to $P_{o p}$, i.e., $P_{s r c}^{\prime}=P_{o p}$. Let $T^{*}$ be the rise time for constant pressure PSSs at the same $P_{s r c}$. The condition that $P_{s r c}$ in a constant flow PSS does not drop below $P_{o p}$ during inflation is then given by: 


$$
P_{s r c} \bar{V}+P_{0} \bar{Q} T^{*}+P_{0}>P_{o p}\left(\bar{V}+1+K_{e x p}\left(P_{o p}-P_{0}\right)\right)
$$

As the average $P_{s r c}$ in constant flow PSSs is always lower than that in constant pressure PSSs, $T_{\text {rise }}>$ $T^{*}$. Hence, if the above condition is satisfied, it is always ensured that $P_{s r c}$ after inflation does not drop below $P_{o p}$, and therefore, $T_{\text {rise }}$ will be similar to that of constant pressure PSSs. If Eq. 8 is not satisfied, $P_{s r c}$ can drop below $P_{o p}$ and the compressor must pressurize both soft actuator and reservoir, which greatly increases $T_{\text {rise }}$.

Such behaviour is seen due to the presence of the reservoir. Since $\dot{P}_{s r c}$ is inversely proportional to $\bar{V}$, dynamics of $P_{s r c}$ for PSSs with large $\bar{V}$ will be slower than those with smaller $\bar{V}$. The reservoir thus acts analogous to a capacitor, and permits rapid bursts of flow to the soft actuator, which can be several times higher than the peak compressor output $\bar{Q}$. Thus, PSSs with large reservoirs can compensate for poor compressor flow capacity. However, this is valid only for intermittent operation, as the flow to the soft actuator has to be replenished by the compressor.

3) Maximum actuation frequency, $f_{\max }$ :

a) Constant Pressure PSSs: We used $T_{\text {rise }}$ and $T_{\text {fall }}$ to calculate $f_{\max }$ for different sets of $P_{\text {src }}, K_{\text {exp }}$, $b_{e q}$ and $P_{o p}$. This frequency is given by,

$$
f_{\text {max }}=\frac{1}{T_{\text {rise }}+T_{\text {fall }}} H z=\frac{1}{T_{\text {Cycle }}} H z
$$

From Eq. 9 and Fig. 3 and 4 , we see that $f_{\max }$ increases with $P_{s r c}, b_{e q}$ and $\bar{C}$ and decreases with $P_{o p}$ and $K_{\text {exp }}$.

In order to achieve cyclic actuation, the valve must be controlled in a repeating and alternating pattern to inflate and deflate the actuator. The ratio of ON time to the OFF time is the valve duty cycle $D_{V}$ (more rigorous definition described in SI Appendix). To achieve $f_{\max }$ given by 9 , the $D_{V}$ required is given by:

$$
D_{V}=\frac{T_{\text {rise }}}{T_{\text {Cycle }}} \times 100 \%
$$

At any frequency higher than that in Eq. 9, the amplitude of pressure oscillation will reduce. Additionally, at $f_{\max }$, duty cycles other than that in Eq. 10 will either lead to overpressure, or reduce the pressure amplitude.

b) Constant Flow PSSs: In constant flow PSSs, as $P_{s r c}$ is not constant, $f_{\text {max }}$ cannot be calculated directly from $T_{\text {fall }}$ and $T_{\text {rise. }}$. While fast inflation can be achieved in intermittent operation, continuous cyclic actuation may not be sustained at the same speed as the compressor may not be able to replenish the air consumed. As a result, $P_{s r c}$ will drop, $T_{\text {rise }}$ will increase and the actuation frequency will reduce. However, if this continues, steady state will be reached such that $T_{\text {rise }}$ does not increase further. At 
the steady state, $f_{\max }$ can be found by applying conservation of mass to the air entering (through the compressor) and leaving (from the soft actuator) the system.

Soft actuator pressure and normalized volume before inflation is $P_{0}$ and 1 respectively. After inflation, they increase to $P_{o p}$ and $\left(1+K_{e x p}\left(P_{o p}-P_{0}\right)\right)$ respectively. The amount of air entering the system during one actuation cycle is $P_{0} \bar{Q} T_{C y c l e} D_{C}$, where $D_{C}$ is the compressor duty cycle. By mass conservation, we have,

$$
P_{0}+P_{0} \bar{Q} D_{C} T_{C y c l e}=P_{o p}\left(1+K_{e x p}\left(P_{o p}-P_{0}\right)\right)
$$

Rearranging,

$$
f_{\text {max }}=\frac{1}{T_{\text {Cycle }}}=\frac{P_{0} \bar{Q} D_{C}}{\left(P_{o p}-P_{0}\right)\left(1+P_{o p} K_{\text {exp }}\right)}
$$

If the system reaches steady state, Eq. 12 predicts the sustainable $f_{\max }$ for any compressor based PSS powering any soft actuator, with a simple arithmetic expression containing only 3 terms: normalized average compressor flow, soft actuator operating pressure, and expansion ratio; irrespective of the other variables. Using $D_{C}=1$ gives the theoretically maximum sustainable $f_{\max }$ for a compressor-based PSS.

In order to achieve this $f_{\max }, \bar{C}$ should be sufficiently large enough to allow the desired flow rate, and $D_{V}$ should be chosen appropriately to allow sufficient time for complete inflation and deflation. This condition is given by,

$$
D_{V} \geq \frac{T^{*}}{T_{\text {Cycle }}} \times 100 \% ; \quad D_{V} \leq 1-\frac{T_{\text {fall }}}{T_{\text {Cycle }}} \times 100 \%
$$

where $T^{*}$ is the rise time for constant pressure PSSs at the same initial $P_{s r c}$. If $\bar{C}$ is insufficient to provide the desired flow rate, either one or both conditions in the above equation will not be satisfied, giving an infeasible solution. In such a case, the steady state will not be reached, and the only solution to achieving the theoretically maximum $f_{\max }$ would be to increase $\bar{C}$.

For both types of PSSs, the air consumed per actuation cycle in standard L is given by:

$$
\text { Air Consumed }=\left(P_{o p}-P_{0}\right)\left(1+P_{o p} K_{\text {exp }}\right) V_{\text {spa }}^{0}
$$

\section{IMPLEMENTATION TO PSS DESIGN}

We validate the model via parameter optimization to select PSS components for an entirely portable soft exosuit. The prototyped PSS was aimed to meet both dynamic performance and PSS specifications. 


\section{A. Design requirements}

We have prototyped a soft exosuit as shown in Fig. 77A, for studying force interactions with the human torso. This exosuit consists of six soft actuators, called SPA-packs [17] (Fig. 8A), capable of producing forces up to $40 \mathrm{~N}$ each. We calculated the dynamic performance requirements of this exosuit for an application to support the wearer's weight in awkward work postures, based on [44]-[48], and a pilot study. These requirements must be met by a portable PSS, which should be compact and lightweight. Table II summarizes these performance requirements and PSS specifications.

\section{B. Parameter optimization and component selection}

Using our mapping results, we calculated the range of values of PSS parameters $f$ and $c$ which would meet performance requirements given in Table III. Using these values, we compared available options for source, valve and pneumatic lines in order to find the optimal set of PSS components as described below:

1) For tubing length $0.5 \mathrm{~m}$ (between valve and SPA-pack), we compared options for standard pneumatic tubing with internal diameters of $1.2 \mathrm{~mm}, 2.5 \mathrm{~mm}, 4 \mathrm{~mm}$ and $5 \mathrm{~mm}$. Using Eq. $\mathrm{S} 10$, we calculated $C$ and $b$ for each, as well as $V_{s p a}^{0}$ and $K_{\text {exp }}$ by adding the tubing dead volume to the SPA-pack internal volume. For our specific case, the tubing volume is much smaller with respect to the SPA-pack volume $(0.079 \mathrm{~L})$. Using a conservative approach, we considered the largest diameter to get $V_{s p a}^{0}=0.083 \mathrm{~L}$ and $K_{\text {exp }}=1.51 V_{\text {spa }}^{0} / \mathrm{MPa}$.

2) For $P_{o p}=0.2 \mathrm{MPa} G$ and the above value of $K_{\text {exp }}$, we used Fig. 4, 5 and Fig. 6 to find predicted values of $T_{\text {rise }}$ for a range of $P_{s r c}, \bar{C}$ and $b_{e q}$. The regions above $T_{\text {rise }}=0.45 \mathrm{~s}$ in Fig 6 correspond to values of $P_{s r c}, \bar{C}$ and $b_{e q}$ which will satisfy the requirements of rise time. Additionally, we predicted $T_{f a l l}$ for our customized SPA-pack, for a range of $\bar{C}$ and $b_{\text {eq }}$, using Fig. 3 and $7 \mathrm{E}$. Using the predicted $T_{\text {fall }}$ and $T_{\text {rise }}$ from the figures, we calculated the acceptable range of values of $P_{s r c}, \bar{C}$ and $b_{e q}$.

3) Next, we compared available valves and fittings, and noted the combinations of valve, tubing and fittings with $\bar{C}$ and $b_{e q}$ lying in the acceptable range.

4) From the desired number of full actuations, we calculated the total amount of air required as 897 standard L by applying Eq. 14. Using this and acceptable range of $P_{s r c}$, we compared available options for the source as follows:

a) Pressure regulated: We compared acceptable options among high pressure cylinders and liquid $\mathrm{CO}_{2}$, which could meet the total air requirement, along with their regulators.

b) Compressor based: We compared acceptable compressors and battery combinations that could provide $P_{s r c}$, the total air requirement and $f_{\max }$ (from Eq. 12). Then we calculated $\bar{V}$ required for simultaneously powering four SPA-packs, using Eq. 8, and compared possible options for reservoir. 
5) For every combination of PSS components that could meet performance requirements, we calculated the total PSS volume and mass. We selected the combination with minimum mass of components, as listed in table $\mathrm{S} 1$. It consists of a battery-powered compressor, two reservoirs, standard tubing and fittings, and six sets of proportional valves with custom manifolds.

6) For the selected components, we used Eq. 12 and 13 to calculate optimal $D_{C}$ and $D_{V}$.

With the optimally selected components, we prototyped and assembled the PSS in the form of a backpack as shown in Fig. 7B. The total mass of the selected PSS components is $1.95 \mathrm{~kg}$, and their optimal flow and control parameters are as listed in Fig. 7D, and highlighted in Fig. 3, 4, 5, 6, 7E.

\section{Comparison with experimental testing}

We tested the prototype while powering a single SPA-pack (Fig. 8A) in two conditions, unloaded and blocked. For both cases, we measured the pressure response ten times on a bench-top. To compare the observed dynamic behaviour with that predicted by our model, we simulated the SPA-pack response in these conditions. For simulating blocked conditions, we took $K_{\exp }=0$.

From Fig. 8B, we observe that the PSS meets desired performance requirements of $T_{\text {rise }}, T_{\text {fall }}$ and $f_{\text {max }}$, and the measured data shows good agreement with simulations. Additionally, Table III shows the peak and root mean squared (RMS) errors between simulation and experimental results. RMS errors for blocked conditions and deflation in unloaded conditions were found to be less than 3.1\%. A slightly higher error (5.2\% RMS, 13.2\% peak) was observed for inflation during unloaded expansion (Fig. 8B), which can be attributed to the non-linear behaviour of the SPA-pack. A higher order model for the pressure-volume relationship along with inclusion of inertial and external forces will be carried out in future studies to better understand these rich dynamics exhibited by soft actuators.

\section{CONCLUSION}

In this study, we presented parameter optimization for design and control of PSSs. This is the first comprehensive study and validation of addressing the requirements of both soft actuator dynamic performance and PSS specifications. The model for soft actuator pressure dynamics proposed in this study goes beyond the scope of currently used fitted models as it captures the effects of the PSS components and soft actuator design parameters. Furthermore, it complements existing studies of soft actuator kinematics and deformation, and can be employed to study and dynamically control soft robot motion and forces. Using this model, we investigated and quantified the effect of ten parameters on soft actuator dynamic performance. In addition, we identified physical limits of achievable dynamic performance for a given set of parameters. This mapping creates an improved understanding of the role of source, valve, pneumatic line and soft actuator design parameters on dynamic performance. Using this mapping, we designed a 
PSS for powering an exosuit with six soft actuators, while minimizing mass of PSS components. Results from experimental testing with this device show good agreement with simulations, thereby validating the proposed approach. By enabling optimized PSSs for robotic applications this work is a step towards customizing soft robotic systems and will help to broaden the scope of soft robots.

\section{ACKNOWLEDGEMENTS}

\section{This work was supported by the Swiss National Science Foundation (SNSF) "START” Project 513956.}

\section{REFERENCES}

[1] Daniela Rus and Michael T Tolley. Design, fabrication and control of soft robots. Nature, 521(7553):467-475, 2015.

[2] Filip Ilievski, Aaron D Mazzeo, Robert F Shepherd, Xin Chen, and George M Whitesides. Soft robotics for chemists. Angewandte Chemie, 123(8):1930-1935, 2011.

[3] Gunjan Agarwal, Nicolas Besuchet, Basile Audergon, and Jamie Paik. Stretchable materials for robust soft actuators towards assistive wearable devices. Scientific reports, 6:34224, 2016.

[4] Yong-Lae Park, Bor-rong Chen, Néstor O Pérez-Arancibia, Diana Young, Leia Stirling, Robert J Wood, Eugene C Goldfield, and Radhika Nagpal. Design and control of a bio-inspired soft wearable robotic device for ankle-foot rehabilitation. Bioinspiration \& biomimetics, 9(1):016007, 2014.

[5] Yong-Lae Park, Jobim Santos, Kevin G Galloway, Eugene C Goldfield, and Robert J Wood. A soft wearable robotic device for active knee motions using flat pneumatic artificial muscles. In Robotics and Automation (ICRA), 2014 IEEE International Conference on, pages 4805-4810. IEEE, 2014.

[6] Harshal Arun Sonar and Jamie Paik. Soft pneumatic actuator skin with piezoelectric sensors for vibrotactile feedback. Frontiers in Robotics and AI, 2:38, 2016.

[7] Hong Kai Yap, Nazir Kamaldin, Jeong Hoon Lim, Fatima Nasrallah, James CH Goh, and Chen-Hua Yeow. A magnetic resonance compatible soft wearable robotic glove for hand rehabilitation and brain imaging. IEEE transactions on neural systems and rehabilitation engineering, 2016.

[8] Michael Wehner, Brendan Quinlivan, Patrick M Aubin, Ernesto Martinez-Villalpando, Michael Baumann, Leia Stirling, Kenneth Holt, Robert Wood, and Conor Walsh. A lightweight soft exosuit for gait assistance. In Robotics and Automation (ICRA), 2013 IEEE International Conference on, pages 3362-3369. IEEE, 2013.

[9] Hong Kai Yap, Benjamin WK Ang, Jeong Hoon Lim, James CH Goh, and Chen-Hua Yeow. A fabric-regulated soft robotic glove with user intent detection using emg and rfid for hand assistive application. In Robotics and Automation (ICRA), 2016 IEEE International Conference on, pages 3537-3542. IEEE, 2016.

[10] Michael T Tolley, Robert F Shepherd, Bobak Mosadegh, Kevin C Galloway, Michael Wehner, Michael Karpelson, Robert J Wood, and George M Whitesides. A resilient, untethered soft robot. Soft Robotics, 1(3):213-223, 2014.

[11] Robert F Shepherd, Filip Ilievski, Wonjae Choi, Stephen A Morin, Adam A Stokes, Aaron D Mazzeo, Xin Chen, Michael Wang, and George M Whitesides. Multigait soft robot. Proceedings of the National Academy of Sciences, 108(51):20400-20403, 2011.

[12] Cagdas D Onal and Daniela Rus. Autonomous undulatory serpentine locomotion utilizing body dynamics of a fluidic soft robot. Bioinspiration \& biomimetics, 8(2):026003, 2013.

[13] Andrew D Marchese, Cagdas D Onal, and Daniela Rus. Autonomous soft robotic fish capable of escape maneuvers using fluidic elastomer actuators. Soft Robotics, 1(1):75-87, 2014.

[14] Ming Luo, Mahdi Agheli, and Cagdas D Onal. Theoretical modeling and experimental analysis of a pressure-operated soft robotic snake. Soft Robotics, 1(2):136-146, 2014. 
[15] Adam A Stokes, Robert F Shepherd, Stephen A Morin, Filip Ilievski, and George M Whitesides. A hybrid combining hard and soft robots. Soft Robotics, 1(1):70-74, 2014.

[16] Mariangela Manti, Taimoor Hassan, Giovanni Passetti, Nicolò D’Elia, Cecilia Laschi, and Matteo Cianchetti. A bioinspired soft robotic gripper for adaptable and effective grasping. Soft Robotics, 2(3):107-116, 2015.

[17] Matthew A Robertson, Hamed Sadeghi, Juan Manuel Florez, and Jamie Paik. Soft pneumatic actuator fascicles for high force and reliability. Soft Robotics, 4(1):23-32, 2017.

[18] Michael Wehner, Ryan L Truby, Daniel J Fitzgerald, Bobak Mosadegh, George M Whitesides, Jennifer A Lewis, and Robert J Wood. An integrated design and fabrication strategy for entirely soft, autonomous robots. Nature, 536(7617):451-455, 2016.

[19] Laura Paez, Gunjan Agarwal, and Jamie Paik. Design and analysis of a soft pneumatic actuator with origami shell reinforcement. Soft Robotics, 3(3):109-119, 2016.

[20] Fionnuala Connolly, Conor J Walsh, and Katia Bertoldi. Automatic design of fiber-reinforced soft actuators for trajectory matching. Proceedings of the National Academy of Sciences, 114(1):51-56, 2017.

[21] Ellen T Roche, Robert Wohlfarth, Johannes TB Overvelde, Nikolay V Vasilyev, Frank A Pigula, David J Mooney, Katia Bertoldi, and Conor J Walsh. A bioinspired soft actuated material. Advanced Materials, 26(8):1200-1206, 2014.

[22] Michael Wehner, Michael T Tolley, Yiğit Mengüç, Yong-Lae Park, Annan Mozeika, Ye Ding, Cagdas Onal, Robert F Shepherd, George M Whitesides, and Robert J Wood. Pneumatic energy sources for autonomous and wearable soft robotics. Soft Robotics, 1(4):263-274, 2014.

[23] Bobak Mosadegh, Panagiotis Polygerinos, Christoph Keplinger, Sophia Wennstedt, Robert F Shepherd, Unmukt Gupta, Jongmin Shim, Katia Bertoldi, Conor J Walsh, and George M Whitesides. Pneumatic networks for soft robotics that actuate rapidly. Advanced Functional Materials, 24(15):2163-2170, 2014.

[24] Deepak Trivedi, Amir Lotfi, and Christopher D Rahn. Geometrically exact models for soft robotic manipulators. IEEE Transactions on Robotics, 24(4):773-780, 2008.

[25] Panagiotis Polygerinos, Zheng Wang, Johannes TB Overvelde, Kevin C Galloway, Robert J Wood, Katia Bertoldi, and Conor J Walsh. Modeling of soft fiber-reinforced bending actuators. IEEE Transactions on Robotics, 31(3):778-789, 2015.

[26] Valentin Falkenhahn, Alexander Hildebrandt, Rüdiger Neumann, and Oliver Sawodny. Model-based feedforward position control of constant curvature continuum robots using feedback linearization. In Robotics and Automation (ICRA), 2015 IEEE International Conference on, pages 762-767. IEEE, 2015.

[27] Andrew D Marchese and Daniela Rus. Design, kinematics, and control of a soft spatial fluidic elastomer manipulator. The International Journal of Robotics Research, 35(7):840-869, 2016.

[28] Andrew D Marchese, Russ Tedrake, and Daniela Rus. Dynamics and trajectory optimization for a soft spatial fluidic elastomer manipulator. The International Journal of Robotics Research, 35(8):1000-1019, 2016.

[29] Cosimo Della Santina, Robert K Katzschmann, Antonio Bicchi, and Daniela Rus. Dynamic control of soft robots interacting with the environment. In IEEE International Conference on Soft Robotics 2018. IEEE, 2018.

[30] Christian Duriez. Control of elastic soft robots based on real-time finite element method. In Robotics and Automation (ICRA), 2013 IEEE International Conference on, pages 3982-3987. IEEE, 2013.

[31] Philip Moseley, Juan Manuel Florez, Harshal Arun Sonar, Gunjan Agarwal, William Curtin, and Jamie Paik. Modeling, design, and development of soft pneumatic actuators with finite element method. Advanced Engineering Materials, 18(6):978-988, 2016.

[32] Andrew D Marchese, Robert K Katzschmann, and Daniela Rus. A recipe for soft fluidic elastomer robots. Soft Robotics, 2(1):7-25, 2015.

[33] Cagdas D Onal and Daniela Rus. A modular approach to soft robots. In Biomedical Robotics and Biomechatronics (BioRob), 2012 4th IEEE RAS \& EMBS International Conference on, pages 1038-1045. IEEE, 2012.

[34] Boran Wang, Andrew McDaid, Morteza Biglari-Abhari, Timothy Giffney, and Kean Aw. A bimorph pneumatic bending actuator by control of fiber braiding angle. Sensors and Actuators A: Physical, 257:173-184, 2017. 
[35] Panagiotis Polygerinos, Zheng Wang, Kevin C Galloway, Robert J Wood, and Conor J Walsh. Soft robotic glove for combined assistance and at-home rehabilitation. Robotics and Autonomous Systems, 73:135-143, 2015.

[36] Bong-Soo Kang, Curt S Kothera, Benjamin KS Woods, and Norman M Wereley. Dynamic modeling of mckibben pneumatic artificial muscles for antagonistic actuation. In Robotics and Automation, 2009. ICRA'09. IEEE International Conference on, pages 182-187. IEEE, 2009.

[37] Takashi Itto and Kiminao Kogiso. Hybrid modeling of mckibben pneumatic artificial muscle systems. In Industrial Technology (ICIT), 2011 IEEE International Conference on, pages 65-70. IEEE, 2011.

[38] Xiangrong Shen. Nonlinear model-based control of pneumatic artificial muscle servo systems. Control Engineering Practice, 18(3):311$317,2010$.

[39] T Nguyen, J Leavitt, F Jabbari, and JE Bobrow. Accurate sliding-mode control of pneumatic systems using low-cost solenoid valves. IEEE/ASME Transactions on mechatronics, 12(2):216-219, 2007.

[40] Peter Beater. Pneumatic drives: System Design, Modelling and Control. Springer, 2007.

[41] Paul G Harris, Garret E O'Donnell, and Tom Whelan. Modelling and identification of industrial pneumatic drive system. The International Journal of Advanced Manufacturing Technology, 58(9-12):1075-1086, 2012.

[42] ISO 6358-1:2013. https://www.iso.org/standard/56612.html, 2013. Accessed: 10-12-2017.

[43] Yoel Shapiro, Alon Wolf, and Kosa Gabor. Bi-bellows: Pneumatic bending actuator. Sensors and Actuators A: Physical, 167(2):484-494, 2011.

[44] Kousei Miura, Hideki Kadone, Masao Koda, Tetsuya Abe, Hiroshi Kumagai, Katsuya Nagashima, Kentaro Mataki, Kengo Fujii, Hiroshi Noguchi, Toru Funayama, et al. The hybrid assistive limb (hal) for care support successfully reduced lumbar load in repetitive lifting movements. Journal of Clinical Neuroscience, 53:276-279, 2018.

[45] Matthew Millard, Manish Sreenivasa, and Katja Mombaur. Predicting the motions and forces of wearable robotic systems using optimal control. Frontiers in Robotics and AI, 4:41, 2017.

[46] Stefano Toxiri, Jesús Ortiz, Jawad Masood, Jorge Fernández, Luis A Mateos, and Darwin G Caldwell. A wearable device for reducing spinal loads during lifting tasks: Biomechanics and design concepts. In Robotics and Biomimetics (ROBIO), 2015 IEEE International Conference on, pages 2295-2300. IEEE, 2015.

[47] Cota Nabeshima, Ko Ayusawa, Conrad Hochberg, and Eiichi Yoshida. Standard performance test of wearable robots for lumbar support. IEEE Robotics and Automation Letters, 3(3):2182-2189, 2018.

[48] Baojun Chen, Lorenzo Grazi, Francesco Lanotte, Nicola Vitiello, and Simona Crea. A real-time lift detection strategy for a hip exoskeleton. Frontiers in neurorobotics, 12:17, 2018.

[49] ISO 6358-1:2013. https://www.iso.org/standard/56612.html, 2013. Accessed: 10-12-2017.

[50] FE Sanville. Two-level compressed air systems for energy saving. In The 7th International Fluid Control Symposium, Bath, England, September, pages 375-383, 1986.

[51] Peter Beater. Pneumatic drives: System Design, Modelling and Control. Springer, 2007.

[52] Paul G Harris, Garret E O'Donnell, and Tom Whelan. Modelling and identification of industrial pneumatic drive system. The International Journal of Advanced Manufacturing Technology, 58(9-12):1075-1086, 2012.

[53] J Eckersten. Simplified flow calculations for pneumatic components. Atlas Copco Air Compendium. Atlas Copco AB, Tukholma, pages 183-192, 1975. 


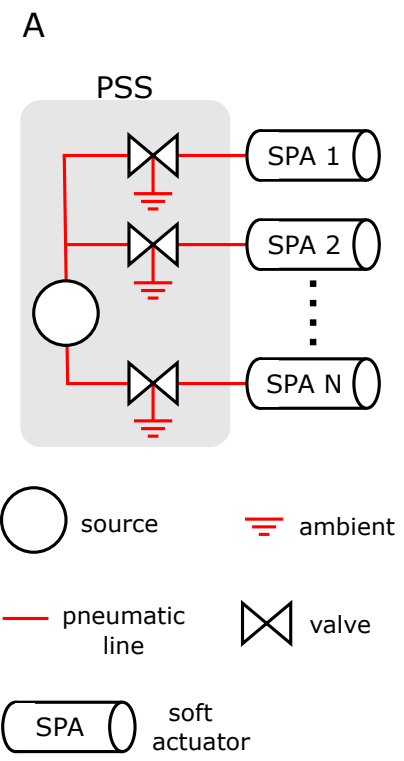

B
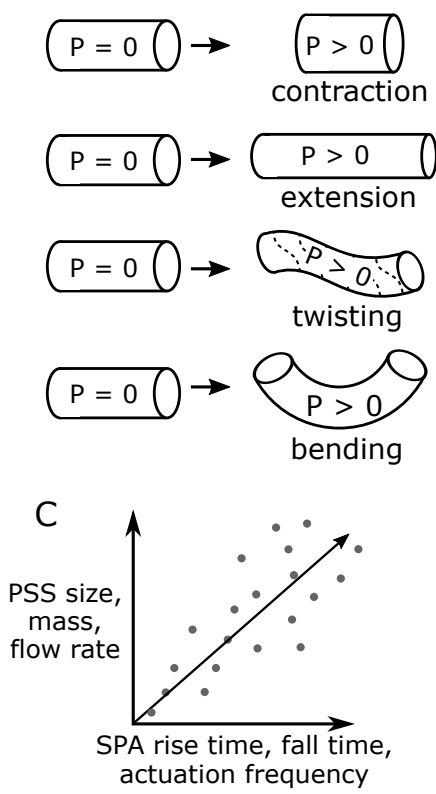

Fig. 1: A pneumatic supply system (PSS) powering soft pneumatic actuators (SPAs) (A) Schematic of a PSS showing the main components, (B) Different actuation patterns achieved by soft actuators when pressurized $(\mathrm{P}>0)$, (C) General trend depicting how improved soft actuator dynamic performance leads to bulkier PSSs.
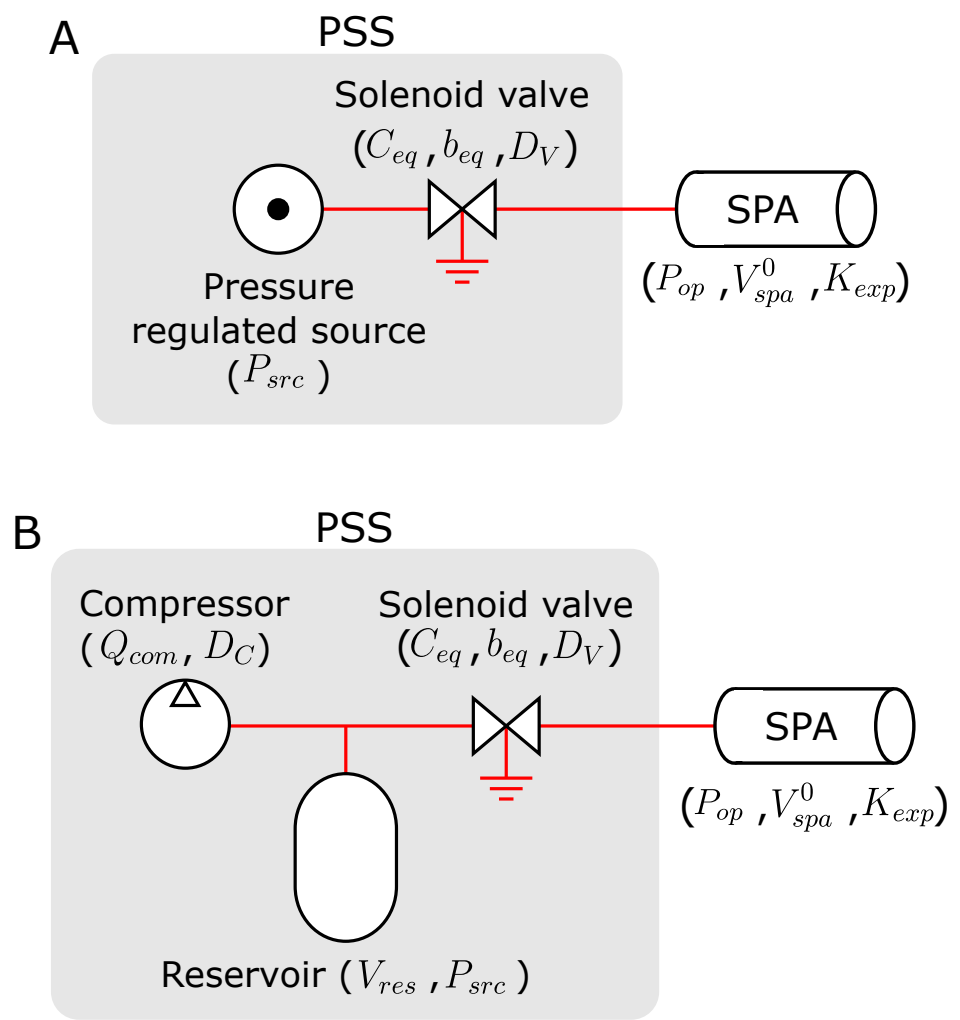

Fig. 2: The two most prominently used PSS configurations: (A) Constant pressure PSSs: PSS with pressure regulator at the source, (B) Constant flow PSSs: Compressor-powered PSS without pressure regulator. 
$b_{e q}$ 0.20

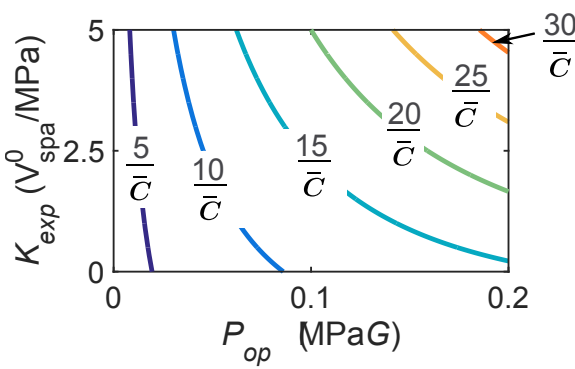

0.49

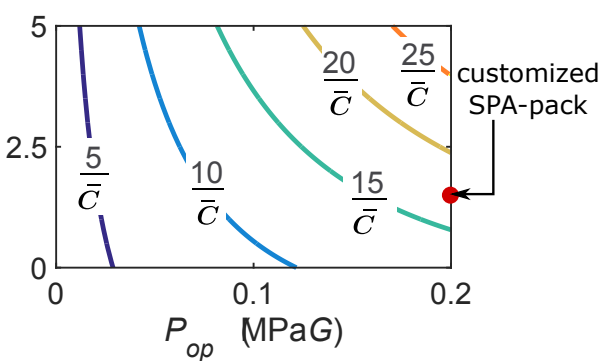

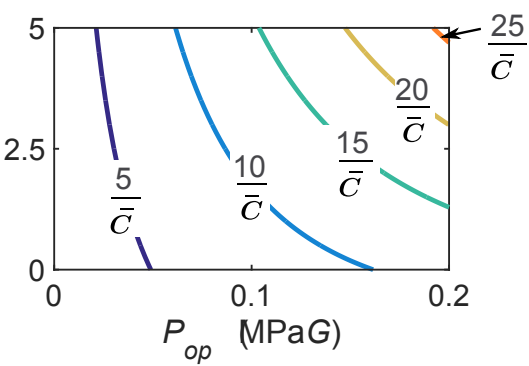

Fig. 3: Fall time, $T_{\text {fall }}$ at varying values of actuator operating pressure, $P_{o p}$, expansion ratio, $K_{\text {exp }}$, and equivalent critical pressure ratio, $b_{e q}$. An axis of increasing $b_{e q}$ is displayed showing the nature of $K_{\exp }$ vs. $P_{o p}$ vs. $T_{f a l l}$ for three values of $b_{e q}$. The fall time in seconds is given by the labels on the contour lines, with $\bar{C}$ expressed in $V_{s p a}^{0} / \mathrm{s} / \mathrm{MPa}$. We observe that $T_{\text {fall }}$ increases with $P_{o p}$ and $K_{\text {exp }}$ and decreases with $\bar{C}$ and $b_{e q}$. The highlighted point corresponds to the SPA-pack (Fig. 8A), with a fall time of 16.4/ $\bar{C}$. At the optimized PSS parameters $\bar{C}=26.89$ and $b_{e q}=0.49$, the predicted fall time is $0.6 \mathrm{~s}$. 

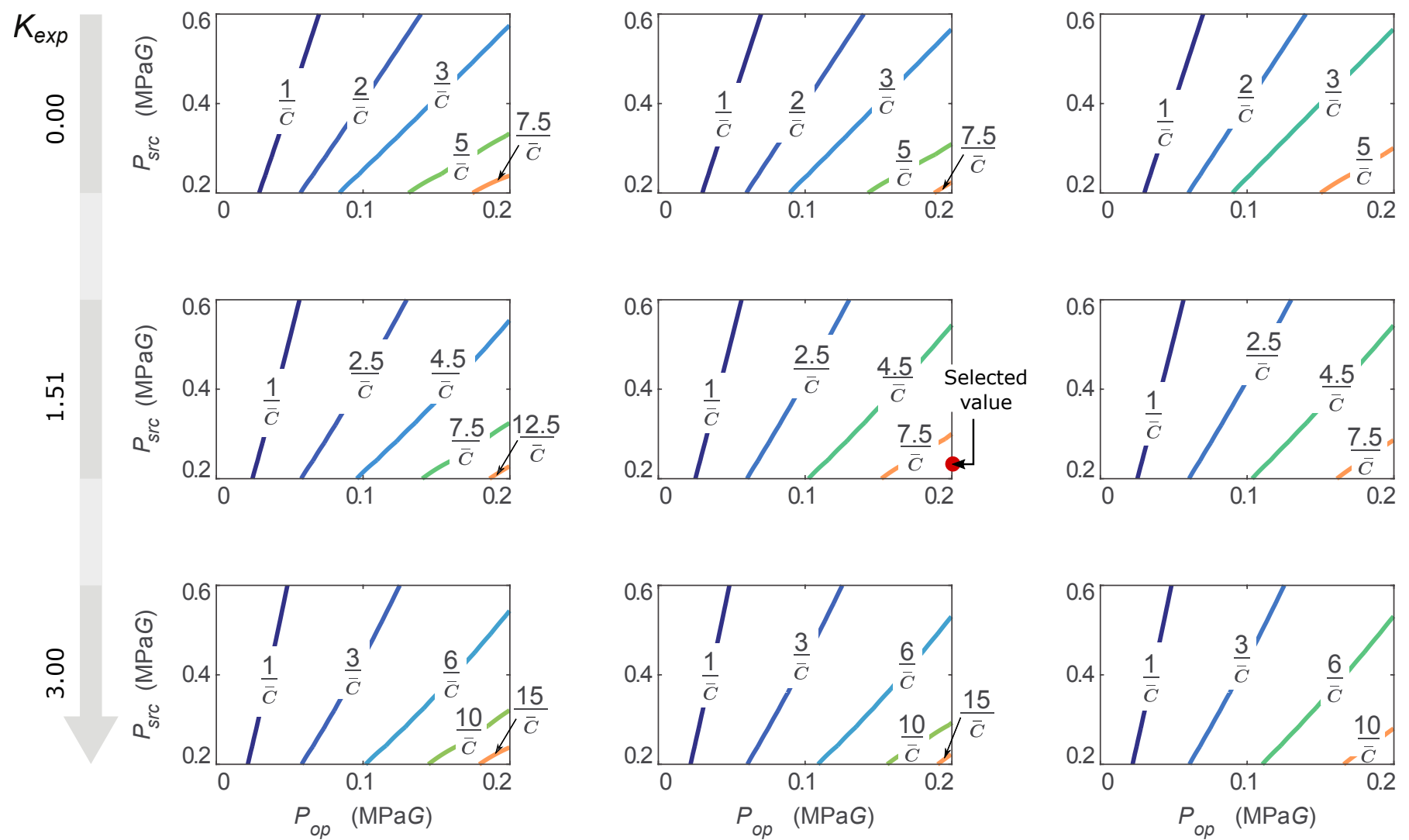

Fig. 4: Rise time, $T_{\text {rise }}$ for constant pressure PSSs at varying values of actuator operating pressure, $P_{o p}$, source pressure, $P_{s r c}$, expansion ratio, $K_{\text {exp }}$ and equivalent critical pressure ratio, $b_{e q}$. Two axes of increasing $b_{e q}$, and $K_{e x p}$ are displayed showing the nature of $P_{s r c}$ vs. $P_{o p}$ vs. $T_{r i s e}$ for three values each of $b_{e q}$, and $K_{\text {exp }}$. The rise time in seconds is given by the labels on the contour lines, with $\bar{C}$ expressed in $V_{s p a}^{0} / \mathrm{s} / \mathrm{MPa}$. We observe that $T_{r i s e}$ increases with $P_{o p}$ and $K_{e x p}$ and decreases with $P_{s r c}, \bar{C}$ and $b_{e q}$. The highlighted point corresponds to our prototype, powering an SPA-pack with $P_{o p}=0.2 \mathrm{MPa} G$ and $K_{\text {exp }}=$ $1.51 V_{s p a}^{0}$. At the optimized PSS parameters $P_{s r c}=0.22 \mathrm{MPa} G, \bar{C}=26.89$ and $b_{e q}=0.49$, the predicted rise time is $0.39 \mathrm{~s}$. 


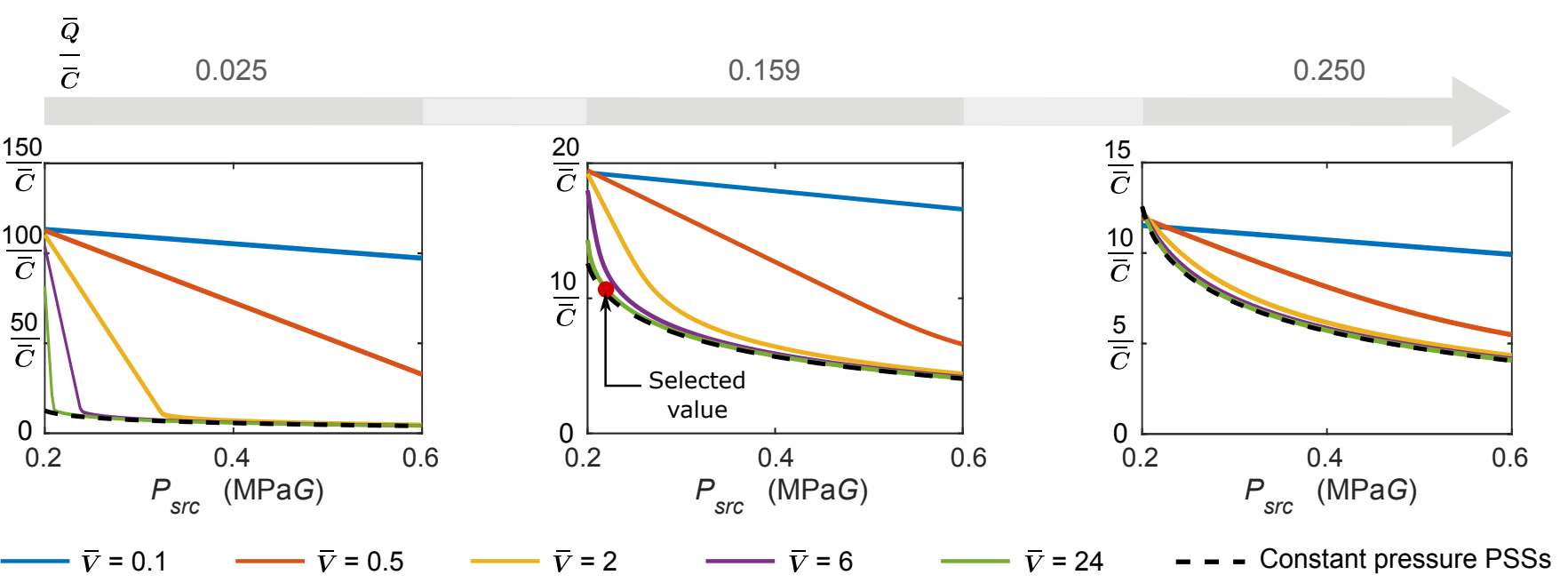

Fig. 5: Rise time, $T_{\text {rise }}$, for constant flow PSSs at varying values of source pressure, $P_{s r c}$, compressor flow output, $\bar{Q}$ and reservoir volumes, $\bar{V}$, for $P_{o p}=0.2 \mathrm{MPaG}, K_{\text {exp }}=1.51 V_{\text {spa }}^{0} / M P a$ and $b_{e q}=0.49$. An axis of increasing $\bar{Q} / \bar{C}$ is displayed showing the nature of $P_{s r c}$ vs. $T_{\text {rise }}$ for three values of $\bar{Q} / \bar{C}$. The rise time in seconds is given by the labels on the Y-axis, with $\bar{C}$ expressed in $V_{s p a}^{0} / \mathrm{s} / \mathrm{MPa}$. We observe that $T_{\text {rise }}$ for constant flow PSSs is very close to that of constant pressure PSSs (dashed curve), for certain values of $P_{s r c}$ and $\bar{V}$. This is because the pressure in the reservoir does not vary much during inflation, leading to a quasi-constant pressure. However, at other values of $P_{s r c}$ and $\bar{V}, T_{\text {rise }}$ is substantially higher. This occurs when $P_{s r c}$ drops below $P_{s p a}$, and the compressor has to pressurize both, reservoir and soft actuator. The transition point at which this occurs can be found using the figure, or from Eq. 8. Here, the highlighted point corresponds to our prototype, with predicted rise time of $10.5 / \bar{C}$ which gives $T_{\text {rise }}=0.39$ s for out selected valve and tubing.

$P_{\text {src }}$

$0.2 \mathrm{MPaG}$

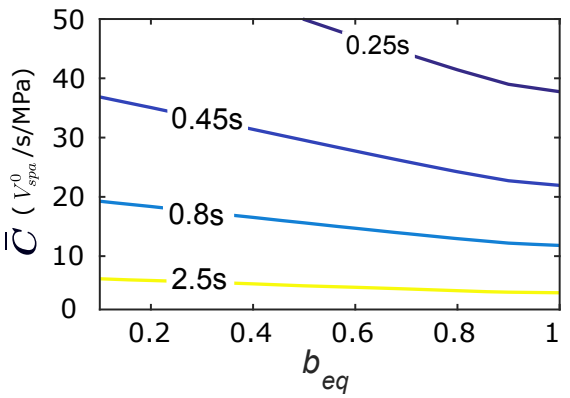

$0.22 \mathrm{MPaG}$

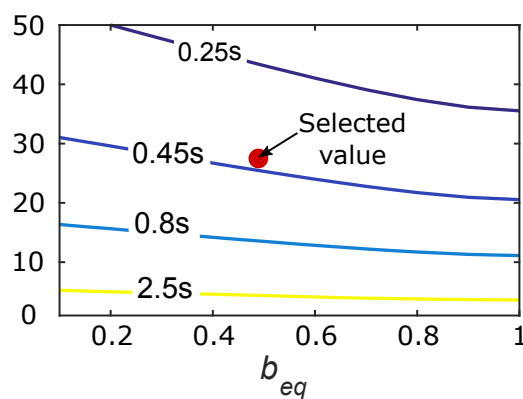

$0.24 \mathrm{MPaG}$

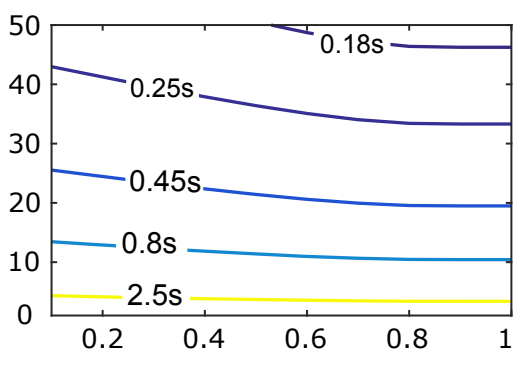

Fig. 6: Predicted rise time for $K_{e x p}=1.51$ and $P_{o p}=0.2 \mathrm{MPa} G$, for varying values of $P_{s r c}, \bar{C}$ and $b_{e q}$. Since the requirement is $T_{\text {rise }}<0.45 \mathrm{~s}$, the selected valve, tubing and fittings should have $\bar{C}$ and $b_{e q}$ lying in the region above the curve $T_{\text {rise }}=0.45 \mathrm{~s}$. For our prototype, the selected components correspond to $P_{\text {src }}=0.22 \mathrm{MPa} G, \bar{C}=26.89$ and $b_{\text {eq }}=0.49$. At these values, we get $T_{\text {rise }}=0.39 \mathrm{~s}$ as shown by the highlighted point. 

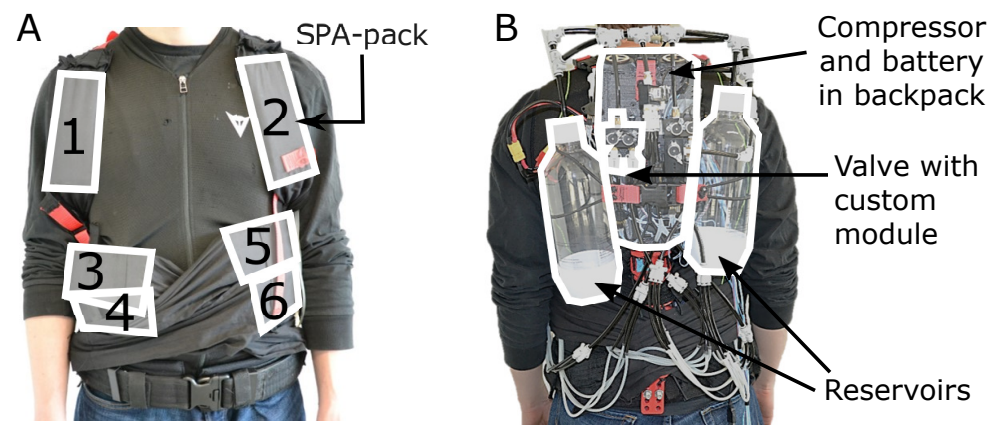

C

PSS

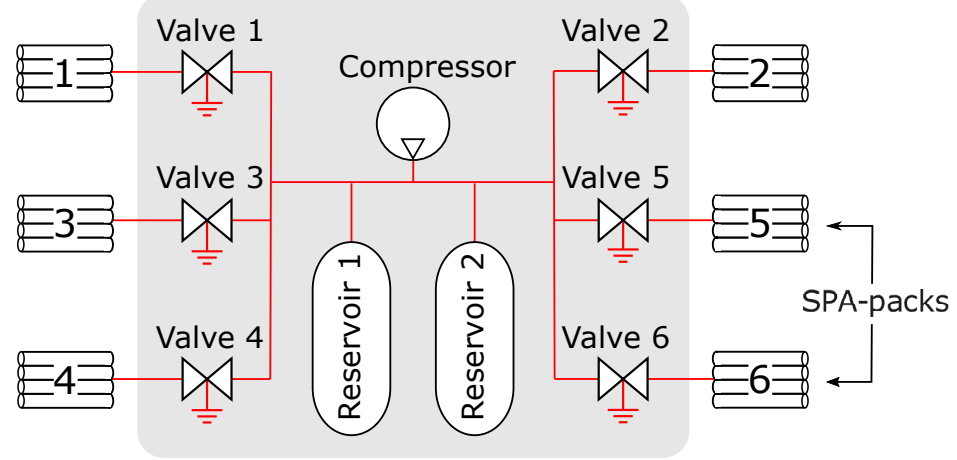

D

E Fall time for $P_{o p}=0.2 \mathrm{MPaG}$;

\begin{tabular}{|c|c|}
\hline \multicolumn{2}{|c|}{ Optimal parameters } \\
\hline \multirow{4}{*}{$f:$} & $\bar{Q}=4.28$ \\
& $\bar{C}=26.89$ \\
& $\bar{V}=24.20$ \\
& $b_{e q}=0.49$ \\
\hline \multirow{4}{*}{$:$} & $P_{s r c}=0.22$ \\
& $D_{C}=54 \%$ \\
& $35 \%<D_{V}<46 \%$ \\
\hline
\end{tabular}

$K_{\text {exp }}=1.51$

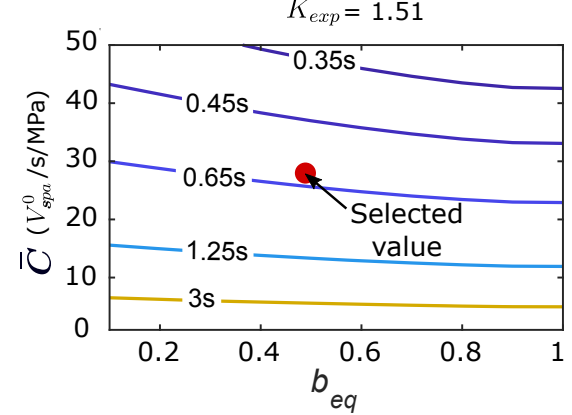

Fig. 7: PSS design via parameter optimization (A) Soft exosuit, which is to be powered by the PSS. It consists of six SPA-packs marked 1 to 6, (B) Prototype of the optimized PSS, fabricated in the form of a backpack. (C) Schematic of the PSS for the exosuit powering 6 SPA-packs, (D) Optimized flow and control parameters required to meet requirements of Table $\amalg$, (E) Predicted fall time fpr $P_{o p}=0.2 \mathrm{MPa} G$ at a range of $\bar{C}$ and $b_{e q}$. The highlighted point corresponds to the selected combination of valve, tubing and fitting for the prototype, with predicted $T_{\text {fall }}=0.6 \mathrm{~s}$. 

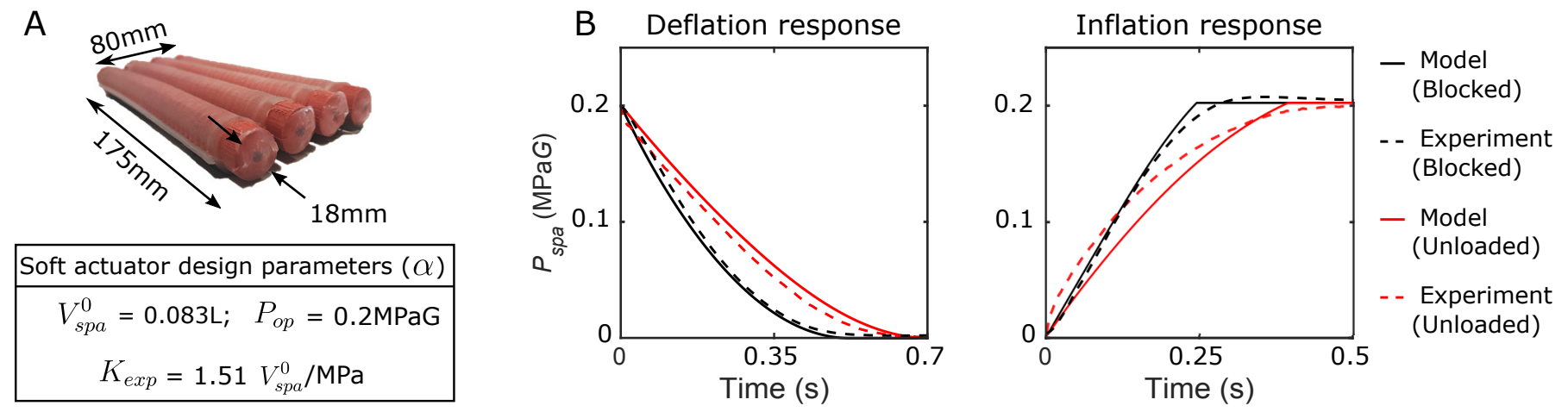

Fig. 8: Validation of PSS parameter optimization via off-board testing of the SPA-pack dynamic performance. (A) The SPA-pack used in the soft exosuit. (B) Comparison of simulations (continuous lines) with measured pressure response (dotted lines) in inflation and deflation, in blocked (black) and unloaded conditions (red). Each test was carried out ten times. $P_{s r c}$ before inflation was $0.22 \mathrm{MPa} G$ and $0.24 \mathrm{MPa} G$ for unloaded and blocked conditions respectively. 
TABLE I: Considered parameters of SPA and PSS

\begin{tabular}{|c|c|c|c|}
\hline \multicolumn{2}{|c|}{ Classification } & Symbol & Description \\
\hline$f$ & $\begin{array}{l}\text { PSS flow } \\
\text { parameters }\end{array}$ & $\begin{array}{c}C_{e q} \\
b_{e q} \\
Q_{c o m} \\
V_{r e s}\end{array}$ & $\begin{array}{c}\text { Equivalent sonic conductance } \\
\text { Equivalent critical ratio } \\
\text { Compressor flow output } * \\
\text { Reservoir volume } *\end{array}$ \\
\hline$c$ & $\begin{array}{l}\text { PSS control } \\
\text { parameters }\end{array}$ & $\begin{array}{l}P_{s r c} \\
D_{V} \\
D_{C}\end{array}$ & $\begin{array}{c}\text { Source Pressure } \\
\text { Valve duty cycle } \\
\text { Compressor duty cycle * }\end{array}$ \\
\hline$\alpha$ & $\begin{array}{l}\text { soft actuator } \\
\text { design } \\
\text { parameters }\end{array}$ & $\begin{array}{c}P_{o p} \\
V_{s p a}^{0} \\
K_{\exp }\end{array}$ & $\begin{array}{l}\text { Operating pressure } \\
\text { Internal volume } \\
\text { Expansion ratio }\end{array}$ \\
\hline
\end{tabular}

*only applicable for configuration $\mathrm{B}$

TABLE II: Design requirements and corresponding achieved values of the fabricated PSS

\begin{tabular}{|c|c|c|}
\hline \multicolumn{2}{|c|}{ Dynamic performance specifications * } & Achieved Values \\
\hline$T_{\text {rise }}$ & $<0.45 \mathrm{~s}$ & $0.42 \mathrm{~s}$ \\
$T_{\text {fall }}$ & $<0.65 \mathrm{~s}$ & $0.59 \mathrm{~s}$ \\
$f_{\max }$ & $>0.9 \mathrm{~Hz}$ & $0.99 \mathrm{~Hz}$ \\
\hline \multicolumn{2}{|c|}{ PSS Specifications } & Achieved Values \\
\hline Overall mass & $<3 \mathrm{~kg}$ & $\mathbf{1 . 9 5} \mathbf{~ k g}$ (optimized) \\
Overall volume & $<0.4 \times 0.3 \times 0.2 \mathrm{~m}$ & $0.3 \times 0.2 \times 0.15 \mathrm{~m}$ \\
Tubing length** & $0.5 \mathrm{~m}$ & $0.5 \mathrm{~m}$ \\
Output channels & 6 & 6 \\
Number of actuations & $>5000$ & $>6000$ \\
\hline
\end{tabular}

*specifications for a single SPA-pack

** from valve to SPA-pack

TABLE III: Comparison of measured pressure response with simulations

\begin{tabular}{|c|c|c|c|c|}
\hline \multirow{2}{*}{} & \multicolumn{2}{|c|}{ RMS Error [\%] } & \multicolumn{2}{c|}{ Peak Error [\%] } \\
\cline { 2 - 5 } & Unloaded & Blocked & Unloaded & Blocked \\
\hline Inflation & 5.2 & 1.6 & 13.2 & 6.0 \\
Deflation & 3.1 & 2.1 & 5.2 & 5.1 \\
\hline
\end{tabular}




\section{SUPPLEMENTARY INFORMATION}

\section{A. Modelling pressure dynamics of PSS}

We classify PSSs into two types: constant pressure PSSs and constant flow PSSs; as described in Fig. 2. For constant pressure PSSs, we have $\dot{P}_{s r c}=0$. For constant flow PSSs, $P_{s r c}$ does not remain constant due to inflow and outflow from the reservoir. Applying the Ideal gas equations and expressing mass flow rate as standard volumetric flow at $P_{0}, T_{0}$, the source pressure dynamics are given by:

$$
\dot{P}_{\text {src }} V_{\text {res }}=\frac{R T_{0}}{M_{\text {air }}} \rho_{\text {air }}\left(Q_{\text {com }}-\operatorname{pos}\left(Q_{\text {spa }}\right)\right)
$$

where $V_{\text {res }}$ is the reservoir volume including dead volume of the tubing and fittings between the compressor and reservoir, $R$ is the Ideal gas constant, $M_{a i r}$ is molar mass of air, $\rho_{\text {air }}$ is air density at $P_{0}, T_{0}, Q_{\text {com }}$ is the mass flow from the compressor expressed in standard $\mathrm{L} / \mathrm{s}$, and $\operatorname{pos}\left(Q_{\text {spa }}\right)$ is the positive mass flow from the actuator, i.e., flow from reservoir to soft actuator, in standard L/s.

Negative actuator flow does not affect $P_{s r c}$, as the air is exhausted to the ambient. For one mole of air at standard conditions, $M_{\text {air }} / \rho_{\text {air }}=V_{0} \times 10^{-6}$, and $P_{0} V_{0} \times 10^{-6}=R T_{0}$, where $V_{0}$ is the volume of one mole of air at $P_{0}, T_{0}$. The $10^{-6}$ term is seen because $V_{0}$ is expressed in L instead of $\mathrm{m}^{3}$. Using these relations in Eq. $\mathrm{S} 1$ we get,

$$
\dot{P}_{\text {src }}=\frac{P_{0}}{V_{\text {res }}}\left(Q_{\text {com }}-\operatorname{pos}\left(Q_{\text {spa }}\right)\right)
$$

The flow $Q_{s p a}$ is through the valve and pneumatic line, i.e. tubing and fittings. To model it, we use ISO 6358 [49]. This is an empirical approximation of the analytical solution of flow through an orifice, and is suitable for modelling gas flow through a variety of pneumatic components as well as through series and parallel combinations [50]-[52]. It describes the flow through a component using two parameters, sonic conductance, $C$, and critical ratio, $b,(0<b<1)$, as shown below:

$$
\Psi= \begin{cases}\sqrt{1-\left(\frac{\frac{P_{\text {Low }}}{P_{\text {High }}}-b}{1-b}\right)^{2},} & \frac{P_{\text {Low }}}{P_{\text {High }}} \geq b \\ 1, & \frac{P_{\text {Low }}}{P_{\text {High }}}<b\end{cases}
$$

where $P_{\text {High }}$ and $P_{\text {Low }}$ are the absolute upstream and downstream pressures respectively; $C$ and $b$ are the sonic conductance and critical ratio respectively of the component, and $\Psi$ is the non-linear flow function.

The nature of flow depends on the ratio of downstream to upstream pressure. If the pressure ratio is below the critical value, $b$, the flow is choked, i.e. air flows at sonic speed, and the flow is only dependent 
on $P_{H i g h}$ and $C$. When pressure ratio is above $b$, the flow is a non-linear function of $b, C$ and $P_{H i g h}$. When the pressure ratio becomes 1, there is no flow due to absence of a pressure differential. While Eq. S3 and S4 define flow for a single component, the combined effect of the various components in the flow path can be modelled with the same set of equations by using the equivalent conductance and critical ratio [51]. Below, we discuss empirical expressions for the elementary combinations of series and parallel, which can be used to model complex PSSs containing several components [51], [53].

- Series combinations: For components connected in series, the equivalent parameters $C_{e q}$ and $b_{e q}$ are calculated in sequence starting from the first component in the direction of flow. First an auxiliary variable, $\epsilon$, is calculated as given by [53]:

$$
\epsilon=\frac{C_{1}}{b_{1} C_{2}}
$$

where indices 1 and 2 correspond to the first and second component respectively in the flow path (in the direction of flow).

The value of $\epsilon$ predicts which component will be choked (sonic flow). If $\epsilon \leq 1$, flow in the first component becomes choked. If $\epsilon=1$, both components have choked flow. If $\epsilon \geq 1$, the second component becomes choked.

Next, the equivalent sonic conductance $C_{1,2}$ of the series connection is given by [51], [53]:

$$
C_{1,2}= \begin{cases}C_{1} & , \epsilon \leq 1 \\ \epsilon C_{2} \frac{\epsilon b_{1}+\left(1-b_{1}\right) \sqrt{\epsilon^{2}+\left(\frac{1-b_{1}}{b_{1}}\right)^{2}}}{\epsilon^{2}+\left(\frac{1-b_{1}}{b_{1}}\right)^{2}} & , \epsilon \geq 1\end{cases}
$$

And the equivalent critical pressure ratio is given by

$$
b_{1,2}=1-C_{1,2}^{2}\left(\frac{1-b_{1}}{C_{1}^{2}}+\frac{1-b_{2}}{C_{2}^{2}}\right)
$$

If there is a third component, the above steps must be repeated by taking $C_{1,2}, b_{1,2}$ and $C_{3}, b_{3}$. The equivalent parameters, $C_{e q}$ and $b_{e q}$ of the entire flow path can be calculated by sequentially following these steps while considering all components in series.

- Parallel combinations: If the components are connected in parallel, the equivalent sonic conductance is given by the empirical relation described by [51], [53]:

$$
C_{e q}=\sum_{i=1}^{n} C_{i}
$$


And the critical ratio is given by,

$$
\frac{C_{e q}}{\sqrt{1-b_{e q}}}=\sum_{i=1} \frac{C_{i}}{\sqrt{1-b_{i}}}
$$

By appropriately using the relations $S 5$ to 59 , we can calculate $C_{e q}$ and $b_{e q}$ for the set of tubing, fittings and valves, as well as more complex networks connecting several components. $C$ and $b$ for valves, tubing and fittings can be generally found from manufacturers' technical manuals, or can be characterized using the guidelines given by ISO 6358 [49]. For the tubing, they can also be calculated using the following empirical relationship as described by Eckersten et. al [51], [53]:

$$
C_{\text {tubing }}=\frac{0.029 D^{2}}{\sqrt{\frac{L}{D^{1.25}}+510}} ; \quad b=\frac{474 C}{D^{2}}
$$

where $L$ and $D$ are the tubing length and inner diameter respectively in $\mathrm{m}$.

\section{B. Normalization}

Eq. 1, 2, 5, S3, S4, S5, S6, S7, S8, S9 and S10 describe soft actuator pressure dynamics. To reduce the dimensionality, we normalize our model with $V_{s p a}^{0}$ and $C_{e q}$. We first normalize equivalent sonic conductance as follows:

$$
\bar{C}=\frac{C_{e q}}{V_{s p a}^{0}}
$$

where $\bar{C}$ is expressed in $V_{s p a}^{0} / \mathrm{s} / \mathrm{MPa}$,

For constant flow PSSs, we additionally have:

$$
\bar{V}=\frac{V_{\text {res }}}{V_{\text {spa }}^{0}}, \quad \bar{Q}=\frac{Q_{\text {com }}}{V_{\text {spa }}^{0}}
$$

where $\bar{V}$ is the normalized reservoir volume expressed in $V_{s p a}^{0}$, $\bar{Q}$ is the normalized compressor flow in $V_{s p a}^{0} / \mathrm{s}$.

Next, we normalize soft actuator pressure dynamics by $\bar{C}$ as:

$$
\begin{gathered}
\left.\dot{P}_{s p a}\right|_{\text {Infl }}=\left(\frac{P_{0} \Psi_{\text {Inf }} P_{s r c}}{1+K_{\text {exp }}\left(2 P_{\text {spa }}-P_{0}\right)}\right) \bar{C} \\
\left.\dot{P}_{\text {spa }}\right|_{\text {Defl }}=\left(\frac{-P_{0} \Psi_{\text {Def }} P_{\text {spa }}}{1+K_{\text {exp }}\left(2 P_{\text {spa }}-P_{0}\right)}\right) \bar{C}
\end{gathered}
$$

where $\Psi_{\text {Inf }}$ and $\Psi_{\text {Def }}$ correspond to the flow function, $\Psi$, during inflation and deflation respectively. For constant pressure PSSs,

$$
\dot{P}_{s r c}=0
$$

And for constant flow PSSs, the normalized source pressure dynamics are given by:

$$
\dot{P}_{s r c}=\left(\frac{P_{0}}{\bar{V}}\left(\bar{Q} / \bar{C}-\Psi_{\text {Inf }} P_{s r c}\right)\right) \bar{C}
$$


Thus, normalized soft actuator pressure dynamics for constant pressure PSSs depend on $P_{\text {src }}, P_{\text {spa }}$, $K_{\text {exp }}$, and $b_{e q}$; and for constant flow PSSs, they additionally depend on $\bar{Q}$ and $\bar{V}$. The effect of $V_{s p a}^{0}$ and $\bar{C}$ is seen as scaling of the dynamics in magnitude and time respectively.

\section{Defining valve duty cycle}

To achieve cyclic actuation, an alternating and repeating control sequence has to be applied to the valve. For the context of this paper, we define two valve states, ON and OFF, as described in Fig. S1. The total ON time includes time of inflation (red), in addition to the time for which the soft actuator stays at $P_{o p}$ (blue). Similarly, OFF time includes the time for deflation (yellow) and the time for which the soft actuator stays at $P_{0}$ (green). Based on these ON and OFF valve states, we define valve duty cycle $D_{V}$ as the ratio of the valve $\mathrm{ON}$ time to the total cycle time ( $\mathrm{ON}+\mathrm{OFF}$ times). Thus, a cyclic actuation will constitute alternating $\mathrm{ON}$ and $\mathrm{OFF}$ states of the valve, in which the variables are valve operation frequency and duty cycle. $f_{\max }$ is achieved when the valve switches state as soon as it reaches one of the extremes $P_{o p}$ or $P_{0}$, i.e., only the red and yellow sections.

While we make an assumption here that $P_{\text {spa }}$ oscillates between $P_{o p}$ and $P_{0}$, in practice, care must be taken to ensure that $P_{s p a}$ does not increase beyond $P_{o p}$ to prevent overpressure, which may damage the soft actuator.

\section{Air consumption}

During deflation, the air in the soft actuator is exhausted to the ambient. Using Eq. 11, the amount of air exhausted during one actuation cycle can be calculated as:

$$
\text { Total Air Consumed }=\left(P_{o p}-P_{0}\right)\left(1+P_{o p} K_{\text {exp }}\right) V_{\text {spa }}^{0}
$$

The total amount of air consumed is simply the product of the above with the number of actuations.

The general expression for air consumed in time $T$ is given by:

$$
\text { Total Air Consumed }=\int_{0}^{T} n e g\left(Q_{\text {spa }}\right) d T
$$

where $\operatorname{neg}\left(Q_{\text {spa }}\right)$ is the negative soft actuator flow, i.e., the exhaust air flow to the ambient during deflation. 


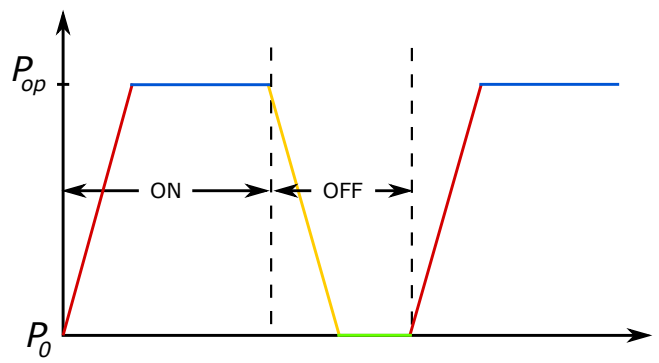

Fig. S1: The different valve states during cyclic actuation. The ON time includes inflation (red), and $P_{s p a}=P_{o p}$ (blue). The OFF time includes deflation (yellow) and $P_{s p a}=P_{0}$ (green). Duty cycle is defined as the ratio of $\mathrm{ON}$ time to the total cycle time (ON + OFF Time). 
TABLE S1: Details of optimally selected components

\begin{tabular}{|c|c|c|}
\hline Component & Description & mass (kg) \\
\hline \multirow{2}{*}{ Compressor } & Double piston compressor, Thomas $G D$ & 0.7 \\
& $\begin{array}{c}\bar{Q}=6.02-7.93\left(P_{\text {src }}-P_{0}\right) \\
\text { Output flow is linear with back pressure, i.e. } P_{s r c}\end{array}$ & \\
\hline Battery & at $P_{\text {src }}=0.22 \mathrm{MPaG}, \quad \bar{Q}=4.28$ & 0.68 \\
\hline Reservoir & Two SodaStream bottles, $1 \mathrm{~L}$ each & 0.06 \\
\hline Valve and & Proportional flow valve, Parker LowPro. Controllable & \multirow{2}{*}{ Turnigy } \\
\hline manifold & sonic conductance with peak value of $C=2.2 L / s / M P a$ & 0.074 each \\
\hline Tubing & Standard tubing, SMC & 0.023 \\
\hline Fittings & Standard fittings, SMC & 0.051 \\
\hline & Overall mass & $\mathbf{1 . 9 5}$ \\
\hline
\end{tabular}

